\title{
STUDY ON THE MALONDIALDEHID LEVEL OF DIABETIC RAT IMPLEMENTED WITH ISOLATE 1.4-BIS- $(3,4,5$-TRIMETOKSI- FENIL)-TETRAHIDRO-FURO $(3,4-C)$ MAHOGANI SEED FURAN (SWIETENIA MACROPHYLLA KING)
}

\author{
Muthmainah'), Bambang Purwanto', Ambar Mudigdo3), \\ Mustofa4) \\ 1)Doctoral Program in Medicine, Universitas Sebelas Maret \\ 2)Department of Internal Medicine, Dr. Moewardi Hospital \\ 3)Department of Anatomic Pathology, Faculty of Medicine, \\ Universitas Sebelas Maret \\ 4)Department of Pharmacology, Faculty of Medicine, \\ Universitas Gadjah Mada
}

\begin{abstract}
Background: In diabetes mellitus (DM), hyperglycemia may increase malondialdehyde (MDA) levels. Isolates 1,4-bis-(3,4,5-trimethoxy-phenyl)tetrahydro-furo $(3,4-c)$ furan from mahogany (Swietenia macrophylla king) can lower blood glucose levels of diabetic rats. This study aimed to examine the blood MDA level of diabetic rats treated with the isolated seeds of mahogany.

Subjects and Method: This was an experimental study conducted at the Laboratory of Center for Food and Nutrition Studies, Inter-University Center, Gadjah Mada University, Yogyakarta. A sample of 36 male Wistar rats, 8-10 weeks old, body weight 200-250 grams, was divided randomly into 6 groups, namely: $\mathrm{KN}$ (normal control), $\mathrm{K}(-)$ (DM without therapy), $\mathrm{K}(+)(\mathrm{DM}+$ glibenclamide $0.45 \mathrm{mg} / \mathrm{kg} \mathrm{BW}$ ), P1, P2, and $\mathrm{P}_{3}$ (DM + isolate of $10 \mathrm{mg} / \mathrm{kgBW}, 20$ $\mathrm{mg} / \mathrm{kg} \mathrm{BW}$, and $40 \mathrm{mg} / \mathrm{kg} \mathrm{BW}$ ). Therapy was given for 21 consecutive days. Streptozotocin $45 \mathrm{mg} / \mathrm{kgBW}$ and nicotinamide $110 \mathrm{mg} / \mathrm{kgBW}$ were used to induce the occurrence of diabetic rats. Examination of blood MDA level was performed at H- (before mouse modelled DM), Ho (when mice were modelled DM but not yet treated), $\mathrm{H}_{7}$ (after 7 days of therapy), H14 (after 14 days of therapy), and H21 (after 21 days of therapy). MDA levels $(\mathrm{nmol} / \mathrm{mL})$ were measured by thiobarbituric acid reacting substances (TBARS) method. Data were analyzed by One Way ANOVA test followed by Tukey HSD and repeated ANOVA test followed by LSD.
\end{abstract}

Results: When $\mathrm{H}-$, mean MDA levels of all groups within normal limits $(<2$ $\mathrm{nmol} / \mathrm{mL})$, ie: $\mathrm{KN} 1.25 \pm 0.16 \mathrm{nmol} / \mathrm{mL}, \mathrm{K}(-) 1.22 \pm 0.14 \mathrm{nmol} / \mathrm{mL}, \mathrm{K}(+) 1.44$ $\pm 0.23 \mathrm{nmol} / \mathrm{mL}, \mathrm{P}_{1} 1.40 \pm 0.14 \mathrm{nmol} / \mathrm{mL}, \mathrm{P} 21.54 \pm 0.07 \mathrm{nmol} / \mathrm{mL}, \mathrm{P}_{3} 1.47 \pm$ $0.17 \mathrm{nmol} / \mathrm{mL}$. When Ho, mean MDA content at $\mathrm{K}(-) 8.83 \pm 0.12 \mathrm{nmol} / \mathrm{mL}, \mathrm{K}$ (+) $8.94 \pm 0.28 \mathrm{nmol} / \mathrm{mL}, \mathrm{P} 19.01 \pm 0.22 \mathrm{nmol} / \mathrm{mL}, \mathrm{P} 29.00 \pm 0.32 \mathrm{nmol} / \mathrm{mL}$, and $\mathrm{P} 39.23 \pm 0.42 \mathrm{nmol} / \mathrm{mL}$ which means higher than normal, while the $\mathrm{KN}$ is still within normal limits, ie $1.42 \pm 0.21 \mathrm{nmol} / \mathrm{mL}$. Within 21 days of treatment, mean MDA levels in each treatment group (K (+), P1, P2, and $\left.\mathrm{P}_{3}\right)$ showed significant decreases over time (Ho to H21). Differences in MDA levels between $\mathrm{Ho}_{\mathrm{H}} \mathrm{H}_{7}, \mathrm{H}_{7}$ and $\mathrm{H}_{14}, \mathrm{H}_{14}$ and $\mathrm{H}_{21}$ showed $\mathrm{p}<0.001$. After 21 days of treatment (at H21) there was a significant difference between MDA and $\mathrm{p}<0.001$, except between pair-wise $\mathrm{KN}$ and $\mathrm{K}(+)$ with $\mathrm{p}=0.724, \mathrm{KN}$ and $\mathrm{P}_{3}$ with $\mathrm{p}=0.865$, and $\mathrm{K}(+)$ and $\mathrm{P}_{3}$ with $\mathrm{p}=1.000$ which means the difference is not meaningful.

Conclusion: Furan Seed isolation therapy is effective for reducing MDA levels. MDA levels of diabetic mice receiving 1.4-bis- (3,4,5-trimethoxy-phenyl) isolate therapy -tetrahydro-furo $(3,4-c)$ furan from mahogany seeds (Swietenia

Mid-International Conference on Public Health, Best Western Premier Hotel, Solo, Indonesia, 18-19 April 2018 |260 https://doi.org/10.26911/mid.icph.2018.05.22 
macrophylla King) $40 \mathrm{mg} / \mathrm{kgBW}$ decreased significantly until levels reached a normal state equivalent to mice receiving glibenclamide therapy.

Keywords: malondialdehyde, mahogany seed, isolate, diabetes

\section{Correspondence:}

Muthmainah. Doctoral Program of Medicine, Universitas Sebelas Maret Surakarta, Jl. Ir.Sutami No. 36 A, 57126, Surakarta, Central Java.

Email: muthmain@gmail.com. Mobile: 08156720402. 